PHYSICAL REVIEW D 96, 069902(E) (2017)

\title{
Publisher's Note: High-energy cosmic ray nuclei from tidal disruption events: Origin, survival, and implications \\ [Phys. Rev. D 96, 063007 (2017)]
}

B. Theodore Zhang, Kohta Murase, Foteini Oikonomou, and Zhuo Li

(Received 21 September 2017; published 28 September 2017)

DOI: 10.1103/PhysRevD.96.069902

This paper was published online on 13 September 2017 without the author's corrections incorporated into the published article. The paper has been corrected as of 21 September 2017. The text is correct in the printed version of the journal. 